\title{
Influencia del régimen de fertilización y del momento de inoculación en la micorrización de Pinus ponderosa en la etapa de vivero
}

\author{
Influence of the fertilization regime and the time of inoculum application in the \\ mycorrhization rate of Pinus ponderosa in nursery
}

\author{
Daniel Baltasar Martíneza, Carolina Barroetaveña ${ }^{a}$, b*, Mario Rajchenberga, b \\ aUniversidad Nacional de la Patagonia S. J. Bosco sede Esquel, Depto. Ingeniería Forestal, \\ Ruta 259 - km 4, Esquel, Chubut, Argentina. \\ *Autor de correspondencia: ${ }^{\text {b}}$ Centro de Investigación y Extensión Forestal Andino Patagónico (CIEFAP), \\ Área Protección Forestal, Ruta 259 km 4, CC. 14 (9200), Esquel, Chubut, Argentina, carolina_barroetavena@yahoo.com \\ Comisión Nacional de Investigaciones Científicas y Técnicas CONICET, Argentina.
}

\begin{abstract}
SUMMARY
This paper has the objective to evaluate mycorrhizal colonization percentages and seedling growth of inoculated Pinus ponderosa seedlings, applying Rhizopogon roseolus spores, three fertilization levels, two inoculation moments (two weeks and 4 months after sowing), and two harvest moments (eight months after sowing and after a two months period under low fertilization, called "lethargy", 10 months after sowing). It was found that the incorporation of the "lethargy" period significantly increased mycorrhization. Both variation in fertilization levels and different inoculation moments did not produce significant differences in mycorrhization percentages, under the traditional production regime.
\end{abstract}

Key words: ectomycorrhizas, ponderosa pine, Rhizopogon roseolus, spore inoculation.

RESUMEN

El presente trabajo tiene como objetivo evaluar la colonización micorrícica y el crecimiento de plantas de Pinus ponderosa inoculadas con esporas de Rhizopogon roseolus, sometidas a tres niveles de fertilización, dos momentos de inoculación (a dos semanas y a cuatro meses posteriores a la siembra), y dos momentos de cosecha diferentes (a ocho meses y a 10 meses de la siembra luego de un período de dos meses con baja fertilización llamado período de letargo). Se observó que la aplicación del período de letargo aumentó significativamente la micorrización. Tanto las variaciones en los niveles de fertilización utilizados como los distintos momentos de inoculación no produjeron diferencias significativas en los porcentajes de micorrización, bajo el esquema de producción tradicional.

Palabras clave: ectomicorrizas, inoculación con esporas, pino ponderosa, Rhizopogon roseolus.

\section{INTRODUCCIÓN}

La precordillera andina de la Patagonia Argentina presenta un gran potencial para el desarrollo de forestaciones con especies exóticas de rápido crecimiento (Laclau 2002), siendo el pino ponderosa (Pinus ponderosa Dougl. ex Laws.) la especie más utilizada con este fin. La creciente actividad forestal en la región hace prever un incremento en la demanda de plantas, y plantea la necesidad de ajustar las técnicas de producción a la obtención de ejemplares de calidad, resistentes a condiciones climáticas adversas, preparados para aprovechar los recursos del sitio de plantación y capaces de resistir el ataque de organismos patógenos del suelo. El estado micorrícico de las plantas tiene un rol determinante en todos estos procesos (Perry et al. 1987). La asociación micorrícica se establece entre las raíces de una planta y hongos específicos. La planta produce fotosintatos que comparte con los hongos, cubriendo sus demandas de carbono, y los hongos contribuyen a incrementar la absorción de agua y minerales (especialmente fósforo) que pasan a la planta, brindando protección física a las raíces, induciendo la ramificación radicular y ofreciendo protección bioquímica contra patógenos del suelo. El resultado es el mejoramiento del prendimiento, de la tasa de crecimiento y de la supervivencia de las plantas, particularmente en hábitats adversos como los expuestos al estrés hídrico (Meyer 1973, Reid 1978, Duddridge et al. 1980, Harley y Smith 1983, Boyd et al. 1986), tal como ocurre en los sitios con aptitud forestal en la Patagonia, que además poseen una biota autóctona típicamente vesículo-arbuscular, sin especies ectomicorrícicas que puedan colonizar Pinaceas (Godoy et al. 1994, Fontenla et al. 1998). 
La producción de plantas forestales en la Patagonia Argentina se ha efectuado tradicionalmente en viveros a raíz desnuda. La adquisición de ectomicorrizas se efectúa normalmente en las camas de siembra gracias al aporte del inóculo ectomicorrícico (EM) proveniente del mantillo utilizado para cubrirlas, o a partir de esporas de hongos transportadas por el viento de plantaciones cercanas (Barroetaveña y Rajchenberg 2003, Barroetaveña 2004). La tasa de micorrización detectada en estos viveros ha sido moderada a baja (Barroetaveña y Rajchenberg 2003). En los últimos años se ha implementado la producción de plantas en invernaderos, utilizando contenedores con sustrato estéril, temperatura controlada y altas dosis de fertilizante químico en el agua de riego (fertiirrigación) para acelerar el ritmo de crecimiento, logrando plantas de excelente morfometría en períodos notablemente menores que la producción tradicional (nueve meses contra dos años). Las composiciones específicas de fertilizante químico se aplican de acuerdo a la etapa de desarrollo en que se encuentra la planta (Enricci et al. 2001) diferenciadas en: germinación, establecimiento, crecimiento rápido y rustificación. Dado que las plantas desarrolladas bajo este sistema no pueden adquirir micorrizas del sustrato en que crecen, es de suma importancia que las prácticas de manejo integral del vivero incluyan la inoculación con hongos EM, efectuando una adecuada selección de las especies a utilizar.

La incorporación de hongos EM se puede realizar utilizando inóculo vegetativo (micelio); sin embargo, los viveros comerciales frecuentemente utilizan esporas como fuente de inóculo (Rincón et al. 2001, 2005, Steinfeld et al. 2003), debido a que éstas no necesitan de una fase de cultivo puro como el inóculo vegetativo, ocupan poco volumen, pueden tolerar períodos de almacenamiento largos, en general son muy abundantes en los esporocarpos y su aplicación es sencilla, ya que se las puede agregar en el agua de riego (Parladé et al. 1996, Rincón et al. 2001). La selección de las especies micorrícicas a utilizar debe considerar la eficacia, la disponibilidad y la logística de obtención del inóculo (Donald 1975). Rhizopogon roseolus (Corda) Th. Fr. (Rhizopogonaceae, Boletales, Basidiomycota) es una especie muy abundante en las plantaciones forestales de Pinus spp. ya establecidas en la Patagonia Argentina (Barroetaveña et al. 2005), que ofrece numerosas ventajas comparativas frente a otras especies EM, pues logra una buena colonización de los sistemas radicales de plantas producidos en maceta y fertiirrigados (Barroetaveña y Rajchenberg 2000, Rincón et al. 2001, 2005), posee alta tolerancia a las condiciones medioambientales adversas (Duñabeitia et al. 2004) y presenta numerosas hifas y rizomorfos que penetran en el suelo maximizando la captura de nutrientes (Donald 1975). Además, fructifica abundantemente presentando altas concentraciones de esporas por cuerpo fructífero; sus esporas poseen una alta tasa de germinación, una gran capacidad de colonizar las raíces con dosis bajas y de mantener su viabilidad luego del almacenamiento a bajas temperaturas
(Theodorou y Bowen 1987, Molina y Trappe 1994, Torres y Honrubia 1994), mientras que el inóculo vegetativo de varias especies del género no ha sido infectivo (Molina 1980, Castellano 1994). Además, numerosos trabajos de inoculación de coníferas con especies del género Rhizopogon han mostrado mejoras en el establecimiento y desarrollo en plantación (Theodorou y Bowen 1970, Theodorou 1971, Momoh 1976, Castellano et al. 1985, Ekwebelam y Odeyinde 1985, Amaranthus y Perry 1989, Castellano 1996). Por todo lo expuesto, $R$. roseolus es una especie potencial atractiva para inocular plantas de Pinus spp. a escala comercial (Chu-Chou y Grace 1990, Parladé et al. 1996, Rincón et al. 2005).

La influencia de altas dosis de fertilizante sobre la colonización micorrícica se ha reportado con diferentes resultados; algunos investigadores afirman que las plantas desarrolladas bajo altas dosis de fertilizante químico no necesitan de una asociación simbiótica para la captación de nutrientes y agua, por lo que la micorrización es pobre en caso de existir (Gagnon et al. 1987, 1988, Hunt 1988, Reitveld et al. 1989, Chakravarty y Chatarpaul 1990, Le Tacon et al. 1997, Smith y Reid 1997); otros afirman que la formación de micorrizas es independiente del nivel de fertilización utilizado (Molina y Chamard 1983, Danielson et al. 1984, Khasa et al. 2001), mientras que Trappe (1977) y Marx (1980) afirman que las distintas asociaciones EM no responden de igual forma a las dietas de fertilizante usadas y que cada caso debe estudiarse por separado.

El momento de aplicación del inóculo EM en estos sistemas de producción acelerada es un tema conflictivo, pues en general se recomienda inocular junto con la siembra para estimular la colonización y dar tiempo al desarrollo de las micorrizas (Marx y Cordell 1990), pero, como se mencionó anteriormente, muchos estudios han demostrado que las altas dosis de fertilizante inhiben el establecimiento de la simbiosis (Le Tacon et al. 1997, Smith y Reid 1997). Si las prácticas de inoculación se realizan durante la fase de rustificación, cuando las dosis de nitrógeno y fósforo son mínimas, puede suceder que el tiempo restante hasta el momento de plantación no sea suficiente para garantizar una buena colonización.

El objetivo principal de este trabajo fue estudiar la asociación micorrícica $R$. roseolus $+P$. ponderosa bajo las condiciones de crecimiento en invernáculo con fertiirrigación, y cómo se establece esta relación bajo diferentes dosis de fertilizante. Algunos estudios reportan que se produce buena micorrización luego de suspender o minimizar la fertilización, aun cuando previamente se aplicaron altas dosis de fertilizantes (Wallander y Nylund 1992, Barroetaveña 2004).

Por lo expuesto, los objetivos particulares planteados para este trabajo son: a) Evaluar el efecto de distintos niveles de fertilización sobre la colonización micorrícica de $P$. ponderosa en vivero utilizando esporas de $R$. roseolus. b) Evaluar el efecto de distintos momentos de aplicación de esporas de $R$. roseolus en la colonización micorrícica de 
plantas de $P$. ponderosa en vivero. c) Evaluar la respuesta de la colonización micorrícica de $R$. roseolus en $P$. ponderosa ante una disminución acentuada en las dosis de fertilizante al final de la etapa de rustificación en vivero (período de letargo). d) Evaluar el efecto de la colonización micorrícica bajo distintos niveles de fertilización y de la aplicación del período de letargo sobre el crecimiento de las plantas.

\section{MÉTODOS}

Para evaluar el efecto del fertilizante sobre la colonización micorrícica y las diferencias de crecimiento entre tratamientos inoculados y no inoculados, se utilizó un diseño factorial con tres niveles de fertilización (alto, medio y bajo) y dos situaciones de inoculación (inoculado a dos semanas de la siembra y control no inoculado para chequear contaminantes y comparar crecimientos), con cinco repeticiones, cada una con tres plantas (experimento 1). Para evaluar el efecto del momento de inoculación y de la aplicación del período de letargo se utilizó también un diseño factorial, con dos momentos de inoculación (a dos semanas y a cuatro meses de la siembra) y dos momentos de cosecha (a ocho meses y a 10 meses de la siembra, luego de un período de letargo), aplicando sólo el régimen de fertilización alto, con cinco repeticiones, cada una con tres plantas (experimento 2).

Las semillas de $P$. ponderosa fueron estratificadas durante 21 días a $4-5^{\circ} \mathrm{C}$, y se esterilizaron superficialmente con una solución de $\mathrm{NaClO} 1 \%$ durante cuatro minutos. La siembra se realizó en macetas plásticas de $250 \mathrm{~cm}^{3}$ de capacidad, con un sustrato compuesto por turba y arena volcánica en relación 1:1 en volumen. La turba se tindalizó mediante tres aplicaciones de vapor a presión atmosférica de 90 minutos cada una, espaciadas entre sí por un lapso de $12 \mathrm{~h}$. La arena se esterilizó en estufa a $120^{\circ} \mathrm{C}$ durante $60 \mathrm{~h}$. Las plantas permanecieron en invernadero sin calefacción, y fueron rotadas periódicamente de ubicación a fin de evitar efectos de borde.

Para las inoculaciones se preparó una suspensión de esporas licuando fructificaciones de $R$. roseolus con agua destilada estéril. La concentración de esporas de la suspensión se determinó mediante un hemocitómetro. Los tratamientos inoculados a las dos semanas de la siembra recibieron dos dosis de $1,5 \times 10^{8}$ esporas por planta (total: $3 \times 10^{8}$ ) espaciadas dos semanas entre sí. Dado que el porcentaje de esporas viables decrece con el tiempo, los tratamientos inoculados a los cuatro meses de la siembra recibieron dos dosis con una concentración 50 veces mayor (dos dosis de $7,5 \times 10^{9}$ esporas por planta; total: $\left.1,5 \times 10^{10}\right)$ de acuerdo a lo recomendado por Torres y Honrubia (1994). Se regó al menos tres veces por semana, dependiendo del contenido de humedad del sustrato. El pH del agua se ajustó en 6,5 mediante $\mathrm{H}_{2} \mathrm{SO}_{4}$ diluido en agua.

La fertilización se aplicó dos veces por semana utilizando fertilizantes químicos hidrosolubles en el agua de riego (cuadro 1) tomando como referencia para el nivel alto la cantidad utilizada en el vivero de producción acelerada de plantas del PAIDER Forestal de la Universidad Nacional de la Patagonia San Juan Bosco - Sede Esquel (Argentina); los niveles medio y bajo correspondieron al 80 y $50 \%$ de las partes por millón agregadas en el tratamiento alto, respectivamente.

Cuadro 1. Composición de los fertilizantes utilizados, duración de cada etapa del esquema de producción y dosis de fertilizante usada en cada aplicación a cada nivel de fertilización en cada etapa.

Fertilizers composition, duration of each stage in the production scheme, and fertilizer doze applied each time to each fertilization level in each stage.

\begin{tabular}{|c|c|c|c|c|c|}
\hline \multirow{2}{*}{ Etapa } & \multirow{2}{*}{$\begin{array}{c}\text { Fertilizante } \\
\text { N/P/K }\end{array}$} & \multirow{2}{*}{$\begin{array}{c}\text { Tiempo } \\
\text { (semanas) }\end{array}$} & \multicolumn{3}{|c|}{ Nivel de fertilización $\left(\mathrm{mg} \mathrm{L}^{-1}\right)$} \\
\hline & & & Alto & Medio & Bajo \\
\hline Germinación & - & 2 & - & - & - \\
\hline Establecimiento & $7 / 40 / 17$ & 6 & $177 \mathrm{P}$ & $141,6 \mathrm{P}$ & $88,5 \mathrm{P}$ \\
\hline $\begin{array}{l}\text { Crecimiento } \\
\text { rápido }\end{array}$ & $20 / 20 / 20$ & 6 & $100 \mathrm{~N}$ & $80 \mathrm{~N}$ & $50 \mathrm{~N}$ \\
\hline Rustificación & $4 / 27 / 38$ & 20 & $140 \mathrm{~K}$ & $112 \mathrm{~K}$ & $70 \mathrm{~K}$ \\
\hline Letargo & $4 / 27 / 38$ & 8 & $70 \mathrm{~K}$ & - & - \\
\hline
\end{tabular}

La longitud de raíces necesarias para medir micorrización se estimó tomando 10 raíces laterales de cada planta, de una submuestra de cinco plantas. Se contabilizó la cantidad de puntas finas micorrizadas con $R$. roseolus y el total de puntas. Mediante la ecuación 1 (Marques de Cantou 1990) se determinó el porcentaje de micorrización de la muestra de raíces laterales, y mediante la ecuación 2 (Marques de Cantou 1990) se determinó el tamaño muestral mínimo (longitud de raíces a medir por planta):

$$
\begin{gathered}
P=\frac{P m}{P m+P n m} \\
t=\frac{z_{\left(1-\frac{\alpha}{2}\right)}^{2} p q}{e^{2}}
\end{gathered}
$$

Siendo:

$P m$ = la cantidad de puntas micorrizadas con $R$. roseolus

$P \quad=$ el porcentaje de puntas micorrizadas con $R$. roseolus

Pnm = la cantidad de puntas no micorrizadas $\operatorname{con} R$. roseolus

$q \quad=$ el porcentaje de puntas no micorrizadas con $R$. roseolus $(1-p)$.

$t=$ el mínimo tamaño muestral a medir por planta.

$e \quad=$ el error máximo admitido.

$z_{(1-} \alpha_{/ 2)}=$ el valor de tabla de distribución normal $\left(\mathrm{Z}_{(95 \%)}\right)$. 
Para efectuar las distintas mediciones, cada planta fue dividida en tres secciones cortándolas a 1 y $15 \mathrm{~cm}$ por debajo del nudo cotiledonar. Para evaluar el porcentaje de micorrización se sortearon raíces al azar de la porción superior del sistema radical (raíz superior) hasta alcanzar el mínimo tamaño muestral obtenido de la ecuación 2; se descartó la porción inferior del sistema radical, ya que presentaba raíces muy largas, arrolladas en el fondo de la maceta, con muy poca ramificación lateral y escasa cantidad de puntas finas. Bajo microscopio estereoscópico se cuantificó la cantidad de puntas micorrizadas con $R$. roseolus y la cantidad total de puntas, y posteriormente se calculó el porcentaje de micorrización por planta, usando la ecuación 1. Para calcular la media del porcentaje de micorrización de cada tratamiento se utilizó la ecuación 3:

$$
\overline{X p}=\frac{\sum_{i=1}^{n} P i}{n}
$$

Siendo

$\overline{X p}=$ la media del porcentaje de micorrización del tratamiento.

$P i=$ el porcentaje de puntas micorrizadas de la i-ésima planta del tratamiento.

$n$ = la cantidad de plantas del tratamiento.

El vástago y el sistema radical de cada planta fueron secados en estufa a $105^{\circ} \mathrm{C}$ durante $48 \mathrm{~h}$ para determinar el peso seco.

Con el fin de evaluar el efecto de los distintos niveles de fertilización sobre la colonización micorrícica se realizó un análisis de varianza de un factor (ANOVA) y comparaciones múltiples (prueba de Tukey) sobre los tratamientos inoculados del experimento 1. El efecto de los distintos momentos de aplicación del inóculo y de la aplicación de un período de letargo se evaluó en el experimento 2 mediante un ANOVA de dos factores, y contrastes (prueba de $\mathrm{T}$, controlando el error tipo I con ajuste de Bonferroni).
La diferencia de tamaño entre las plantas inoculadas y las no inoculadas se analizó en el experimento 1, usando el peso seco de la raíz superior, el peso seco del vástago y el peso seco total como variables de respuesta, aplicando en cada caso un ANOVA de dos factores y contrastes (prueba de $\mathrm{T}$, controlando el error tipo I con ajuste de Bonferroni) entre los tratamientos inoculado y no inoculado en cada nivel de fertilización. El efecto de la aplicación del período de letargo sobre el tamaño de las plantas se analizó en el experimento 2 usando las tres variables de respuestas antes mencionadas, mediante ANOVA de dos factores y contrastes (prueba de T, controlando el error tipo I con ajuste de Bonferroni) entre momentos de cosecha para cada momento de inoculación. Además se evaluó el efecto de los distintos niveles de fertilización sobre el tamaño de las plantas en el experimento 1, usando las mismas variables de respuesta antes mencionados, mediante ANOVA de dos factores y contrastes (prueba de $\mathrm{T}$, controlando el error tipo I con ajuste de Bonferroni) entre los niveles de fertilización para cada situación de inoculación. En todos los casos se utilizó un nivel de significación del 95\%, la normalidad de los datos se determinó mediante la prueba de Shapiro-Wilk y la igualdad de varianzas utilizando la prueba de Levene, ambos con un nivel de significación del 95\%. Se utilizó el paquete estadístico SPSS versión 11.5 para Windows.

\section{RESULTADOS}

Efecto de los niveles de fertilización sobre la colonización micorrícica. Los distintos niveles de fertilización utilizados no modificaron significativamente la colonización micorrícica de $R$. roseolus sobre el $P$. ponderosa bajo el esquema tradicional de producción de plantas, sin aplicar período de letargo $(P=0,52)$, con el valor de micorrización más alto $(38,6 \%)$ en el nivel de fertilización bajo (cuadro 2 ). Cabe destacar que se detectó la presencia de ectendomicorrizas del tipo E-strain (Barroetaveña 2004) colonizando abundantemente las plantas inoculadas.

Cuadro 2. Comparación de las medias de porcentaje de micorrización, peso seco del vástago, de la parte superior de la raíz y del total entre los distintos niveles de fertilización para cada situación de inoculación (experimento 1)*.

Comparison between mean mycorrhization percentage, stem, upper root portion and total dry weight means among different fertilization levels under each inoculation situation (experiment 1$)^{*}$.

\begin{tabular}{cccccc}
\hline \multirow{2}{*}{ Inoculación } & Nivel de & Micorrización $(\%)$ & PS vástago & PS raíz sup. & PS total \\
\cline { 3 - 6 } & fertilización & Media $\pm S$ & Media $\pm \mathrm{S}$ & Media $\pm \mathrm{S}$ & Media $\pm \mathrm{S}$ \\
\hline \multirow{3}{*}{ No inoculado } & Alto & 0 & $1,874 \pm 0,181 \mathrm{~ns}$ & $1,888 \pm 0,429 \mathrm{~ns}$ & $5,780 \pm 0,605 \mathrm{~ns}$ \\
& Medio & 0 & $1,818 \pm 0,244 \mathrm{~ns}$ & $1,974 \pm 0,405 \mathrm{~ns}$ & $5,540 \pm 0,487 \mathrm{~ns}$ \\
& Bajo & 0 & $1,548 \pm 0,212 \mathrm{~ns}$ & $1,508 \pm 0,181 \mathrm{~ns}$ & $4,680 \pm 0,212 \mathrm{~ns}$ \\
\hline \multirow{2}{*}{ Inoculado } & Alto & $30,6 \pm 16,75 \mathrm{~ns}$ & $1,994 \pm 0,276 \mathrm{a}$ & $1,860 \pm 0,611 \mathrm{~ns}$ & $5,973 \pm 0,748 \mathrm{a}$ \\
& Medio & $31,6 \pm 6,69 \mathrm{~ns}$ & $1,922 \pm 0,259 \mathrm{a}$ & $1,994 \pm 0,373 \mathrm{~ns}$ & $5,747 \pm 0,431 \mathrm{ab}$ \\
& Bajo & $38,6 \pm 9,31 \mathrm{~ns}$ & $1,546 \pm 0,100 \mathrm{~b}$ & $1,482 \pm 0,230 \mathrm{~ns}$ & $4,687 \pm 0,353 \mathrm{~b}$ \\
\hline
\end{tabular}

*PS: peso seco; S: desviación estándar; raíz sup: parte superior de la raíz. Contrastes con ajuste de Bonferroni.

Letras diferentes indican diferencias significativas a nivel $P=0,05$; ns: no significativo. 
Efecto de los distintos momentos de aplicación de las esporas y de la incorporación del período de letargo sobre la colonización micorrícica. El momento de aplicación de las esporas (a dos semanas y a cuatro meses de la siembra) no afectó significativamente el porcentaje de micorrización de las plantas (para momento de inoculación, $P=0,437$ ), mientras que la aplicación del período de letargo sí tuvo un efecto estimulador significativo en la micorrización (para momento de cosecha $P=0,022$ ). Hay poca evidencia de que el efecto de la aplicación del período de letargo varíe según el momento de inoculación (para la interacción $P=0,131$ ). Cuando las plantas fueron inoculadas a los cuatro meses de la siembra, la colonización micorrícica media fue significativamente más alta en plantas sometidas a período de letargo (media de micorrización $=48,2 \%$, cuadro 3 ) con un aumento promedio del $21,8 \%$ respecto de las que no fueron sometidas a período de letargo $(P=0,01,95 \%$ intervalo de confianza $=6,0$ a $37,6 \%$ ), constituyendo el valor de micorrización más alto obtenido en ambos experimentos. En el caso de las plantas inoculadas a las dos semanas de la siembra, el efecto fue en el mismo sentido (cuadro 3), con un aumento promedio en la colonización micorrícica de 5\% cuando se aplicó el período de letargo, aunque no significativo estadísticamente $(P=0,512)$.

Cuadro 3. Comparación entre los porcentajes medios de micorrización \pm desviación estándar en los diferentes momentos de cosecha para cada momento de inoculación (experimento 2).

Comparison between mean mycorrhization percentages \pm standard deviation from different harvest moments for each inoculation moment (experiment 2).

\begin{tabular}{lccc}
\hline $\begin{array}{c}\text { Momento de } \\
\text { inoculación }\end{array}$ & $\begin{array}{c}\text { Cosecha a } \\
8 \text { meses }\end{array}$ & $\begin{array}{c}\text { Cosecha a } \\
10 \text { meses }\end{array}$ & $\begin{array}{c}\text { Diferencia } \\
\text { entre medias }\end{array}$ \\
\hline A 2 semanas & $30,6 \pm 16,8$ & $35,6 \pm 8,0$ & $-5,0 \mathrm{~ns}$ \\
A 4 meses & $26,4 \pm 4,1$ & $48,2 \pm 14,0$ & $-21,8^{*}$ \\
\hline
\end{tabular}

Contrastes con ajuste de Bonferroni; *diferencia de medias significativa, $P=0,05$; ns: no significativo.

Respuesta del crecimiento de las plantas a la micorrización. No se encontraron diferencias significativas en el peso seco entre plantas inoculadas y los controles no inoculados del experimento 1 bajo ninguno de los tres niveles de fertilización (cuadro 4). Esto se repitió para el peso seco del vástago (inoculación, $P=0,366$ ), para el peso seco de la raíz superior (inoculación, $P=0,938$ ) y para el peso seco total (inoculación, $P=0,626$ ). Tampoco se encontraron diferencias significativas en el peso seco entre plantas sometidas y no sometidas a período de letargo bajo cada momento de inoculación en el experimento 2. Esto se repitió para el peso seco del vástago (cosecha, $P=0,450$ ), de la raíz superior (cosecha, $P=0,408$ ) y para el peso seco total (cosecha, $P=0,241$ ) (datos no mostrados).
Por el contrario, la disminución en las dietas de fertilizantes se correspondió con una disminución en el peso seco de las plantas (cuadro 2) significativo para el peso seco del vástago (fertilización, $P=0,001$ ), para el peso seco de la raíz superior (fertilización, $P=0,028$ ) y para el peso seco total (fertilización, $P=0,004$ ). Los contrastes mostraron diferencias significativas para el peso seco del vástago entre tratamientos inoculados bajo alta y baja fertilización de 0,448 g $(P=0,011,95 \%$ intervalo de confianza $=0,09$ a 0,806$)$ y bajo media y baja fertilización de $0,376 \mathrm{~g}(P=0,037,95 \%$ intervalo de confianza $=0,018$ a 0,734$)$, y también para el peso seco total entre tratamientos inoculados bajo alta y baja fertilización de $1,28 \mathrm{~g}(P=0,034,95 \%$ intervalo de confianza $=0,077 \mathrm{a}$ 2,499) (cuadro 2).

Cuadro 4. Comparación entre medias de peso seco del vástago, de la parte superior de la raíz y del total entre plantas inoculadas y no inoculadas bajo cada nivel de fertilización (experimento 1 )*.

Comparison between stem, upper root portion and total dry weight means between inoculated and non inoculated plants under each fertilization level (experiment 1)*.

\begin{tabular}{lccc}
\hline \multirow{2}{*}{$\begin{array}{c}\text { Nivel } \\
\text { fertilización }\end{array}$} & \multicolumn{3}{c}{ Diferencia de medias (n-i)* } \\
\cline { 2 - 4 } & PS raíz sup. & PS vástago & PS total \\
\hline Alto & 0,280 & $-0,120$ & $-0,194$ \\
Medio & $-0,020$ & $-0,104$ & $-0,204$ \\
Bajo & 0,026 & 0,002 & $-0,004$ \\
\hline
\end{tabular}

* n-i = no inoculado - inoculado. PS: peso seco; raíz sup: parte superior de la raíz. Contrastes con ajuste de Bonferroni; en todos los casos la diferencia de medias fue no significativa a nivel $P=0,05$.

\section{DISCUSIÓN}

En relación al efecto de los distintos niveles de fertilización sobre la colonización micorrícica, los valores de micorrización encontrados son inferiores al 38,6\% en todos los tratamientos, indicando que la micorrización es inhibida incluso utilizando el $50 \%$ de las dosis de fertilizante empleadas en los viveros comerciales de este tipo. Estos resultados coinciden con lo reportado por varios autores (Molina y Chamard 1983, Danielson et al. 1984 y Khasa et al. 2001). Steinfeld et al. (2003), trabajando con la misma asociación árbol-hongo, reportaron $8 \%$ de colonización micorrícica durante el período bajo fertirriego, que luego superaron con un año de trasplante en vivero a raíz desnuda, logrando $28 \%$ de colonización al momento de la extracción. Se observa, sin embargo, una tendencia negativa (aunque no significativa estadísticamente) entre las variables estudiadas, que se manifiesta como un aumento en el porcentaje de micorrización a medida que disminuye el nivel de fertilización (cuadro 2). Otras experiencias 
de inoculación con esporas de $R$. roseolus en viveros de contenedores con fertilización (Parladé et al. 1996, 2001, Rincón et al. 2005) han logrado altos porcentajes de micorrización, pero usando dosis y/o frecuencias de aplicación de fertilizantes inferiores a las usadas en este trabajo. La aparición de morfotipos contaminantes tipo E-strain, originados por Ascomycetes y hallados comúnmente en viveros a raíz desnuda y plantaciones de $P$. ponderosa en la Patagonia Argentina (Barroetaveña 2004), sugiere que son competitivos frente a otras especies ectomicorrícicas, y que son altamente contaminantes, resistiendo altas dosis de fertilizantes.

La introducción del período de letargo muestra un efecto estimulador significativo de la micorrización en las plantas inoculadas. Estos resultados corroboran los estudios de Barroetaveña (2004) y de Wallander y Nylund (1992) para la asociación $R$. roseolus $+P$. ponderosa bajo las condiciones de crecimiento estudiadas, y aparecen como una alternativa atractiva frente a la opción de reducir sustancialmente el régimen de fertilización, con la consecuente pérdida de crecimiento, a fin de lograr la micorrización. La factibilidad técnica y económica de incorporar el período de letargo deberá evaluarse en cada caso, ajustando los esquemas de aplicación de fertilizantes para llegar a tiempo, con plantas bien micorrizadas, a la temporada de plantación, previendo asimismo el acopio de inóculo en los momentos de fructificación del hongo, de forma que se encuentre disponible al momento de ser aplicado.

En relación al momento de aplicación de las esporas, se observa que las plantas inoculadas a los cuatro meses presentaron una menor colonización que las inoculadas a las dos semanas (diferencias no significativas) al ser evaluadas a los ocho meses, situación que se invierte luego del período de letargo. Esto podría deberse a que las esporas aplicadas a los cuatro meses se vieron favorecidas para germinar y colonizar las raíces por la baja en el nivel de fertilización durante el período de letargo, mientras que las esporas aplicadas a las dos semanas de la siembra pudieron haber germinado durante la etapa de fertilización intensa sin condiciones propicias para establecer la simbiosis, o haber perdido poder germinativo o bien haber sido lavadas de las macetas por los sucesivos riegos.

Analizando las variaciones en el tamaño de las plantas, no es extraño que las características morfométricas de las plantas inoculadas sean similares a las de las no inoculadas, ya que en ambientes con abundancia de nutrientes como los fertiirrigados, donde se proveen todos los elementos nutritivos necesarios para el óptimo crecimiento, las micorrizas dejan de ser un factor importante para el desarrollo. Sin embargo, hay que tener en cuenta en esta comparación que los valores de colonización micorrícica alcanzados en los experimentos nunca superaron el 50\%. Por otra parte, la disminución en los niveles de fertilización ocasionó notorias pérdidas en el tamaño de las plantas sin favorecer significativamente la micorrización.

Futuros estudios deberían evaluar detalladamente la competencia de las especies fúngicas contaminantes en estos ambientes, e incorporar la inoculación con otros hongos micorrícicos presentes en la región, como Suillus luteus (L. ex Fr.) S. F. Gray y Hebeloma mesophaeum (Pers.: Fr.) Quél., que en trabajos anteriores no fueron exitosas (Barroetaveña 2004).

La alternativa explorada en este trabajo, consistente en aplicar altos niveles de fertilización hasta la rustificación y luego extenderla ocho semanas aplicando niveles muy bajos de fertilizante (período de letargo), apareció como mucho más eficiente que la disminución en los niveles de fertilización durante las etapas de establecimiento, crecimiento rápido y rustificación. En este sentido, dado que la micorrización no fue estimulada por la baja en los niveles de fertilización, que sí se correspondieron con pérdidas significativas en el tamaño de las plantas, es recomendable la fertiirrigación con la dosis de nutrientes necesaria para el óptimo desarrollo de las plantas. Por otra parte, la incorporación del período de letargo mostró ser efectiva en plantas inoculadas a cuatro meses de la siembra, aumentando significativamente los niveles de micorrización. Entonces, en un esquema de producción de plantas que incorpore el período de letargo se recomienda inocular las mismas antes de ingresar al período de rustificación (a cuatro meses de la siembra) ya que de esta manera se logra mayor micorrización. En caso de que no sea factible la incorporación del período de letargo sería conveniente inocular junto con la siembra de las semillas, tal como recomiendan Marx y Cordell (1990), o al principio de la etapa de establecimiento, donde existe un gran desarrollo del sistema radical. De este modo se minimizan los riesgos de pérdida de viabilidad del inóculo dado que, por un lado, el poder germinativo de las esporas disminuye con el tiempo (Torres y Honrubia 1994) y por otro su conservación en buenas condiciones suele ser dificultosa, aún bajo refrigeración, debido a la actividad microbiana que tiende a fermentarlo.

Si bien la micorrización no redundó en un aumento en el tamaño de las plantas en la etapa de vivero, el viverista debe incorporar prácticas de inoculación adecuadas para garantizar la supervivencia y el buen crecimiento de las plantas en el sitio de forestación, que es donde la micorrización muestra sus efectos.

\section{AGRADECIMIENTOS}

Al Centro de Investigación y Extensión Forestal Andino Patagónico (CIEFAP) por permitir el uso de los materiales y equipamiento del laboratorio de Fitopatología. A los Ing. Juan Enricci y Diego Massone, y al Sr. Eduardo Núñez, por la información brindada y por permitir el uso de las instalaciones del vivero del PAIDER Forestal de la Universidad Nacional de la Patagonia San Juan Bosco. A los tres árbitros que contribuyeron con sus comentarios al mejoramiento del manuscrito final. 


\section{REFERENCIAS}

Amaranthus MP, DA Perry. 1989. Interaction effects of vegetation type and Pacific Madrone soil inocula on survival, growth and mycorrhizal formation of Douglas fir. Can. J. For. Res. 19: 550-556.

Barroetaveña C. 2004. Estudio de las ectomicorrizas de plantines y plantaciones de pino ponderosa (Pinus ponderosa Doug. ex Laws.) y pino oregón [Pseudotsuga menziesii (Mirb) Franco] en la región Andino Patagónica. Tesis Doctoral. San Carlos de Bariloche, Argentina. Universidad Nacional del Comahue. 225 p.

Barroetaveña C, M Rajchenberg. 2000. Estado micorrítico y micorrización controlada de plantines de pino ponderosa y pino oregón en viveros forestales de la región andinopatagónica. Informe final PIA 13/97. Secretaría de Agricultura, Pesca y Alimentación (SAGPyA), Argentina. 81 p.

Barroetaveña C, M Rajchenberg. 2003. Las micorrizas y la producción de plántulas de pino ponderosa en la Patagonia Argentina. Bosque 24 (1): 3-15.

Barroetaveña C, M Rajchenberg, E Cazares. 2005. Mycorrhizal fungi in Pinus ponderosa introduced in Central Patagonia (Argentina). Nova Hedw. 80: 453-464.

Boyd R, RT Furbank, DJ Read. 1986. Ectomycorrhizae and water relation of trees. In Gianinazzi-Pearson V, S Gianinazzi eds. Proceedings of the $1^{\text {st }}$ European Symposium on Mycorrhizae. Dijon, Francia. p. 140-145.

Castellano M. 1994. Current status of outplanting studies using ectomycorrhiza-inoculated forest trees. In Pfleger FL, RG Linderman eds. Mycorrhizae and plant health. St. Paul, Minnesota, Estados Unidos. APS Press. p. 261-281.

Castellano M. 1996. Outplanting performance of mycorrhizal inoculated seedlings. In Mukerji KG ed. Concepts in Mycorrhizal Research. Holanda. Kluwer Academic Publishers. p. 223-301.

Castellano M, JM Trappe, R Molina. 1985. Inoculation of container grown Douglas fir seedlings with basidiospores of Rhizopogon vinicolor and $R$. colossus: effects of fertility and spore application rate. Can. J. For. Res. 15: 10-13.

Chakravarty P, L Chatarpaul. 1990. Effect of fertilization on seedling growth, ectomycorrhizal symbiosis, and nutrient uptake in Larix laricina. Can. J. For. Res. 20: 245-248.

Chu-Chou M, L.J Grace. 1990. Mycorrhhizal fungi of radiata pine seedlings in nurseries and trees in forests. Soil. Biol. Biochem. 22: 959-966.

Danielson R, S Vissar, D Parkinson. 1984. The effectiveness of mycelial slurries of mycorrhizal fungi for the inoculation of container-grown jack pine seedlings. Can. J. For. Res. 14: $140-142$.

Donald DGM. 1975. Mycorrhizal inoculation for pines. South African Forestry Journal 92: 27-29.

Duddridge JA, A Malibari, DJ Read. 1980. Structure and function of mycorrhizal rhizomorphs with special reference to their role in water transport. Nature 287: 834-836.

Duñabeitia MK, S Hormilla, J Garcia-Plazaola, K Txarterina, U Arteche, JM Becerril. 2004. Differential responses of three fungal species to environmental factors and their role in the mycorrhization of Pinus radiata D. Don. Mycorrhiza 14: 11-18.

Ekwebelam SA, MA Odeyinde. 1985. Field response of Pinus species inoculated with ectomycorrhizal fungi in Nigeria. In
Molina R ed. $6^{\text {th }}$ North American Conference on Mycorrhiza. Corvallis, Oregon, Estados Unidos. p. 220.

Enricci J, G Alday, D Massone. 2001. Producción de plantines en contenedores. Acta de las VI jornadas de viveristas forestales de la Patagonia, 15 y 16 de noviembre de 2001. Esquel, Argentina. 5 p.

Fontenla S, R Godoy, P Rosso, M Havrylenko. 1998. Root associations in Austrocedrus forests and seasonal dynamics of arbuscular mycorrhizas. Mycorrhiza 8: 29-33.

Gagnon J, CG Langlois, JA Fortin. 1987. Growth of containerized jack pine seedlings inoculated with different ectomycorrhizal fungi under a controlled fertilization schedule. Can. J. For. Res. 17: 840-845.

Gagnon J, CG Langlois, JA Fortin. 1988. Growth and ectomycorrhizae formation of containerized black spruce seedlings as affected by nitrogen fertilization, inoculum type, and symbiont. Can. J. For. Res. 18: 922-929.

Godoy R, R Romero, R Carrillo. 1994. Estatus micotrófico de la flora vascular en bosques de coníferas del sur de Chile. Rev. Chilena Hist. Natural 67: 209-220.

Harley JL, SE Smith. 1983. Mycorrhizal Symbiosis. Nueva York, Estados Unidos. Academic Press. 605 p.

Hunt GA. 1988. Effect of controlled-release fertilizers on formation of mycorrhizae and growth of container-grown Engelmann spruce. Proceedings of the Combined Western Forest Nursery Council and Intermountain Nursery Association meeting. USDA Forest Service. 8 p. Research Note RM-167.

Khasa P, L Sigler, P Chakravarty, BP Dancik, L Erickson, D Mc Curdy. 2001. Effect of fertilization on growth and ectomycorrhizal development of container-grown and bare-root nursery conifer seedlings. New Forests 22(3): 179-197.

Laclau P. 2002. La forestación en la Patagonia y el cambio climático. Publicaciones de Economía Forestal, Instituto Nacional de Tecnología Agropecuaria (INTA)-Agencia Alemana de Cooperación Técnica (GTZ). Bariloche, Argentina. 55 p. Comunicación Técnica $\mathrm{N}^{\circ} 6$.

Le Tacon F, D Mousain, J Garbaye, D Bouchard, JL Churin, C Argillier, JM Amirault, B Genere. 1997. Mycorhizes, pépinières et plantations forestières en France. Rev. For. Fr. 49: 131-154.

Marques de Cantou MJ. 1990. Probabilidad y estadística para ciencias químico-biológicas. México D.F. Mc Graw-Hill. $460 \mathrm{p}$.

Marx DH. 1980. Ectomycorrhiza fungus inoculations: a tool for improving forestation practices. In Mikola P. ed. Tropical Mycorrhiza Research. Estados Unidos. Oxford University Press. p. 13-71

Marx DH, CE Cordell. 1990. Development of Pisolithus tinctorius ectomycorrhizae on Loblolly pine seedlings from spores sprayed at different times and rates. USDA Forest Service. 4 p. Research Note SE-356.

Meyer FH. 1973. Distribution of ectomycorrhiza in native and man made forests. In Marks GC, TT Kozlowsky eds. Ectomycorrhizae, their ecology and physiology. Nueva York, Estados Unidos. Academic Press. p. 87-105.

Molina R. 1980. Ectomycorrhizal inoculation of containerized western conifer seedlings. Pacific North West and Range Experimental Station, USDA-Forest Service. Estados Unidos. 5 p. Research note PNW-357.

Molina R, J Chamard. 1983. Use of the ectomycorrhizal fungus Laccaria laccata in forestry II: Effects of fertilizer forms and levels on ectomycorrhizal development and growth of container-grown Douglas-fir and ponderosa pine seedlings. Can. J. For. Res. 13: 89-95. 
Molina R, JM Trappe. 1994. Biology of the ectomycorrhizal genus Rhizopogon I: Host associations, host specificity and pure culture syntheses. New. Phytol. 126: 653-675.

Momoh Z. 1976. Synthesis of mycorrhiza on Pinus oocarpa. Ann. Appl. Biol. 82: 221-226.

Parladé J, M Cohen, J Doltra, J Luque, J Pera. 2001. Continuous measurements of stem-diameter growth response of Pinus pinea seedlings mycorrhizal with Rhizopogon roseolus and submitted to two water regimes. Mycorrhiza 11: 129-136.

Parladé J, J Pera, IF Alvarez. 1996. Inoculation of containerized Pseudotsuga menziesii and Pinus pinaster seedlings with spores of five species of ectomycorrhizal fungi. Mycorrhiza 6: 237-245.

Perry D, R Molina, M Amaranthus. 1987. Mycorrhizae, mycorrhizospheres, and reforestation: current knowledge and research needs. Can. J. For. Res. 17: 929-940.

Reid CPP. 1978. Mycorrhizae and water stress. In Riedacker A, MJ Gagnaire-Michard eds. Proceedings of the IUFRO Symposium on Root Physiology and Symbiosis, Nancy, Francia. p. 392-408.

Reitveld WJ, RA Sharp, MF Kienzler, RK Dixon. 1989. Development of ectomycorrhizae on container-grown European larch. Tree Plant. Notes 70: 12-17.

Rincón AM, IF Álvarez, J Pera. 2001. Inoculation of containerized Pinus pinea $\mathrm{L}$. seedlings with seven ectomycorrhizal fungi. Mycorrhiza 11(6): 265-271.
Rincón AM, J Parladé, J Pera. 2005. Effect of ectomycorrhizal inoculation and the type of substrate on mycorrhization, growth and nutrition of containerized Pinus pinea L. seedlings produced in a commercial nursery. Ann. For. Sci. 62: 1-6.

Steinfeld D, MP Amaranthus, E Cazares. 2003. Survival of ponderosa pine (Pinus ponderosa Dougl. ex Laws.) seedlings outplanted with Rhizopogon mycorrhizae inoculated with spores at the nursery. Journal of Arboriculture 29: 197-207.

Smith SE, D Reid. 1997. Mycorrhizal Symbiosis, $2^{\mathrm{a}}$ ed. Cambridge, Inglaterra. Academic Press. 605 p.

Theodorou C. 1971. Introduction of mycorrhizal fungi into soil by spore inoculation of seed. Aust. For. 35: 17-22.

Theodorou C, GD Bowen. 1970. Mycorrhizal responses of radiata pine in experiments with different fungi. Aust. For. 34: 183-191.

Theodorou C, GD Bowen. 1987. Germination of Basidiospores of Mycorrhizal Fungi in the rhizosphere of Pinus radiata D. Don. New Phytologist 106(2): 217-223.

Torres P, M Honrubia. 1994. Basidiospore viability in stored slurries. Mycorrizal Research 98(5): 527-530.

Trappe J. 1977. Selection of fungi for ectomycorrhizal inoculation in nurseries. Annu. Rev. Phytopathol. 15: 203-222.

Wallander H, JE Nylund. 1992. Effects of excess nitrogen and phosphorus starvation on the extramatrical mycelium of ectomycorrhizas of Pinus sylvestris L. New Phytologist 120(4): 495-503. 